\title{
Accuracy Assessment for Measuring Surface Figures of Large Aspheric Mirrors
}

\author{
Young-Soo Kim*, Ki-Beom Ahn, and Kwijong Park \\ Korea Astronomy 8 Space Science Institute, Daejeon 305-348, Korea \\ II Kweon Moon and Ho-Soon Yang \\ Korea Research Institute of Standards and Science, Daejeon 305-340, Korea
}

(Received May 19, 2009 : revised June 17, 2009 : accepted June 18, 2009)

\begin{abstract}
At the time that the Keck-I 10m telescope was constructed in 1993, the era of Very Large Telescopes (VLTs) was opened. Now thirteen VLTs are in operation, and the largest of the monolithic mirrors is $8.4 \mathrm{~m}$ in diameter. Such monolithic mirrors are mostly aspheric and require high accuracies on the surface figures, reaching up to the diffraction limit. At present, next generation telescopes, Giant telescopes, are being developed. One is the GMT (Giant Magellan Telescope) whose size is 25.4 $\mathrm{m}$ in diameter. The primary mirror consists of seven segments figuring elliptical shapes on the surface. The surrounding six segments are off-axis and the edges are steep, as the fast focal ratio is adopted. It means that testing of the mirrors is a challenging task. In this paper, testing methods for the GMT primary mirror are reviewed, and accuracy of measuring devices is assessed. Results and discussions follow.
\end{abstract}

Keywords: Telescope, Mirror, Surface, Accuracy, Aspheric

OCIS codes : (110.6770) Telescopes; (120.3180) Interferometry; (120.3940) Metrology; (220.4840) Optical testing

\section{INTRODUCTION}

Since Hans Lippershey invented the first refracting telescope in 1608 [1] and the first astronomical telescope by Galileo Galilei appeared on the following year, discovery and understanding in astronomy have advanced rapidly. After Sir Isaac Newton developed the first reflecting telescope, making larger telescopes become possible with ease.

Starting from Mt. Palomar's 200 inch telescope in 1949, the era of $4 \mathrm{~m}$ class large telescopes was opened. Since then, after 50 years, $8 \mathrm{~m}$ class very large telescopes (VLTs) appeared, starting from the Keck telescope ${ }^{1}$ in 1993. Soon after more than a dozen VLTs were constructed and are being operated at Chile, Hawaii, Canary islands, America, etc. There are two types of telescopes according to the constitution of the primary mirror. The first type

\footnotetext{
*Corresponding author: ykim@kasi.re.kr
}

1) http://www.keckobservatory.org

2) http://www.eso.org has a single, monolithic mirror like that of a conventional telescope, but with diameter around $8 \mathrm{~m}$ or less. The second type has a primary mirror which consists of several dozens of small mirrors, whose diameter is between $1 \mathrm{~m}$ and $2 \mathrm{~m}$ and whose shape is usually hexagonal.

TABLE 1 shows optical characteristics of several VLTs, each of which has a monolithic primary mirror. The telescope types are Ritchey-Chrétien (RC), Cassegrain, or Gregorian, which means that the mirrors are figured to parabola, hyperbola, or ellipse on either convex or concave surface. Such aspheric mirror surfaces are more difficult to produce than spherical ones.

Those very large telescopes require high accuracy on the image size reaching the diffraction limits. In case of the Gemini telescopes, $50 \%$ of the energy $\left(\theta_{50}\right)$ should fall within a circle of 0.1 arcsec at the wavelength of $2.2 \mu \mathrm{m}$ [2]. The image size of ESO (European Southern Observatory) $\mathrm{VLTs}^{2}$ should be 0.038 arc-seconds in the 1.5 arc-minutes field of view. Requiring such high accuracy means that the telescopes, including the mirrors, should 
TABLE 1. Characteristics of very large telescopes with monolithic primary mirror.

\begin{tabular}{c|c|c|c|c|c}
\hline \hline & Gemini & ESO VLT & Subaru & LBT & Magellan \\
\hline No of telescopes & 2 & 4 & 1 & 2 & $2.4 \mathrm{~m}$ \\
\hline Diameter & $8.1 \mathrm{~m}$ & $8.2 \mathrm{~m}$ & $\mathrm{RC}$ & Cassegrain & Gregorian \\
\hline Type & $\mathrm{RC}$ & $\mathrm{RC}$ & 1.8 & 1.14 & -1.0 \\
\hline $\mathrm{f} / \#$ Primary & 1.8 & 1.8 & & 0.23 & -1.0 \\
\hline Conic const & -1.003756 & -1.004616 & $0.17\left(\theta_{50}\right)$ & FWHM & \\
\hline $\begin{array}{c}\text { Image size } \\
\text { [arcsec] }\end{array}$ & $\begin{array}{c}0.1\left(\theta_{50}\right) \\
\text { at } 2.2 \mu \mathrm{m}\end{array}$ & 1.5 arcmin & & In one structure & \\
\hline Remarks & & & &
\end{tabular}

TABLE 2. Basic concept data of GSMTs.

\begin{tabular}{c|c|c|c|c}
\hline \hline Name & $\begin{array}{c}\text { Diameter } \\
(\mathrm{m})\end{array}$ & $\begin{array}{c}\text { Optical } \\
\text { system }\end{array}$ & $\begin{array}{c}\text { Expected } \\
\text { Completion } \\
(\text { year })\end{array}$ & Participants \\
\hline GMT & $\begin{array}{c}25 \\
(7 \times 8.4 \mathrm{~m})\end{array}$ & Gregorian & 2018 & $\begin{array}{l}\text { USA : Carnegie Institution of Washington, Harvard Univ., } \\
\text { Smithsonian Institution, Univ. Arizona, Texas Univ. at } \\
\text { Austin, Texas A\&M Univ. } \\
\text { Korea : KASI }\end{array}$ \\
\hline TMT & $\begin{array}{c}\text { Australia : Australia Nat'l Univ., AAL (Astronomy Australia } \\
\text { Limited) }\end{array}$ \\
\hline E-ELT & $\begin{array}{c}(492 \\
\text { segments })\end{array}$ & RC & 2017 & $\begin{array}{l}\text { USA : California Institute of Technology, Univ. of California } \\
\text { Canada }\end{array}$ \\
\hline
\end{tabular}
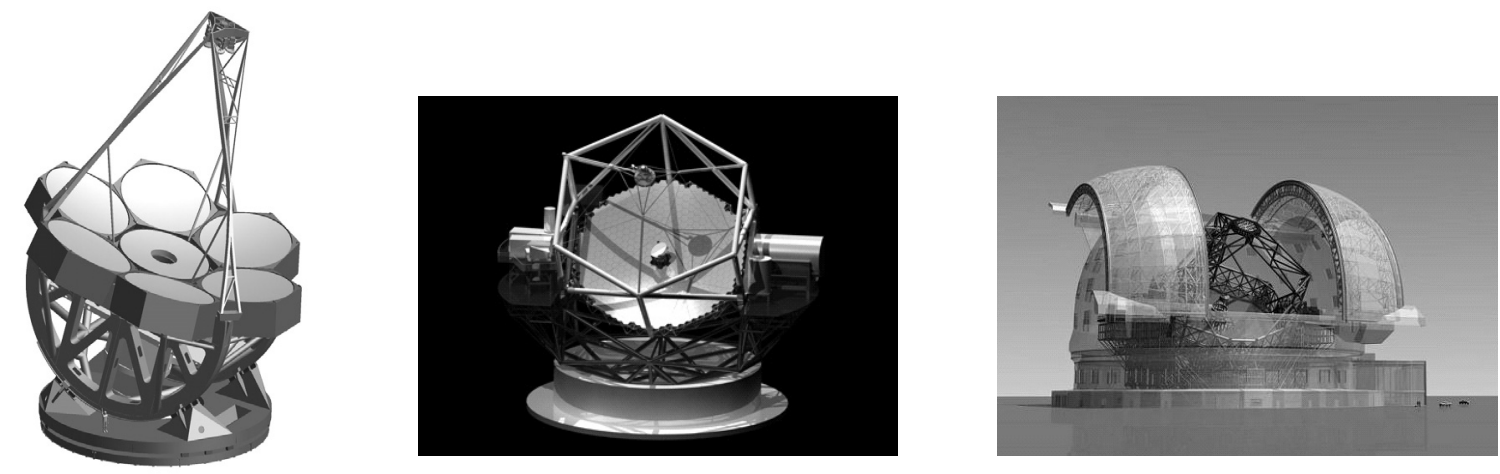

FIG. 1. The three GSMTs: from left GMT, TMT, and E-ELT.

be made very precisely. In order to produce mirrors precisely, test methods should be very accurate. Otherwise, it cannot be judged whether the mirror is produced precisely or not.

After producing the $8 \sim 10 \mathrm{~m}$ VLTs, the next generation telescopes are being developed. Those are three or more times bigger than VLTs and are called GSMTs (Giant Segmented Mirror Telescopes). There are three GSMTs

3) http://www.gmto.org

4) http://www.tmt.org presently being developed, GMT ${ }^{3}$ (Giant Magellan Telescope), TMT $^{4}$ (Thirty Meter Telescope), and E-ELT (European-Extremely Large Telescope). TABLE 2 presents the conceptual data of the GSMTs. GMT inherits the Gregorian type of $6.5 \mathrm{~m}$ Magellan telescopes, and both primary and secondary mirrors have elliptical surfaces. TMT is RC type and E-ELT has a five mirror scheme.

FIG. 1 shows the conceptual drawing of the GSMTs. The primary mirror of the GMT consists of seven $8.4 \mathrm{~m}$ mirror segments, whereas the others consist of several hundreds of hexagonal shaped small mirrors. Those tele- 
scopes are being developed competitively, and aim for similar completion years. Korea officially joins the development of GMT from year 2009.

\section{TESTING METHODS OF LARGE ASPHERIC OFF-AXIS MIRRORS}

Large telescopes are required to have better image quality than smaller telescopes. Though adaptive and active optics can correct the degradation of the image quality caused by inaccurate figure of the telescope mirrors, the optical system should be as perfect as possible, so that deformed mirror figures can be corrected simply and easily.

Testing methods for large mirrors are not very different from those for small mirrors in principle, but there are more things to consider. Large telescopes require high accuracy reaching up to the diffraction limit, therefore

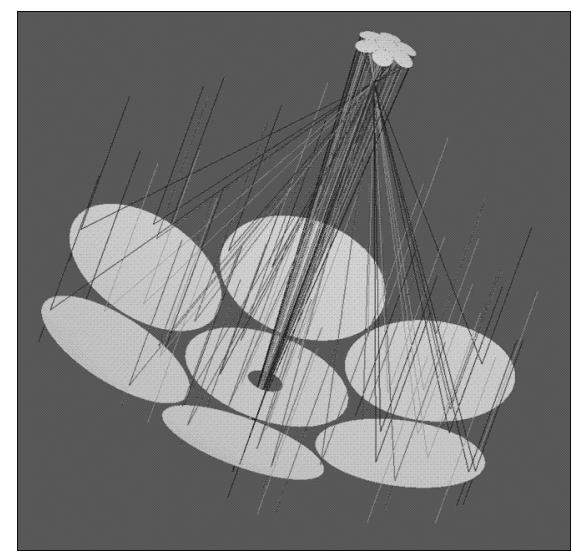

FIG. 2. Optical layout of the GMT optics. It is composed of seven primary and matching secondary mirrors. more precise tests should be performed. The area of the mirror being tested becomes larger and the size can cause difficulty in testing as well. Bigger space is needed and the detector should have a big enough field of view together with sufficient resolution. Environmental factors such as air flow, thermal change of the atmosphere, and ground vibration, should also be considered and controlled carefully [3].

In order to measure the figure errors of large aspheric mirrors, several methods have been developd. In order to compensate the aspheric beam, a null corrector or a CGH (Computer Generated Hologram) has been adopted $[4,5,6]$. Using two wavelengths with phase-shifting interferometry $[7,8,9,10,11]$ or directly measuring aspheric surfaces by profilometry $[6,12]$ were also developed. When it is difficult to test the whole area at once, methods of testing local areas and stitching the results were also developed $[13,14,15]$. Detailed descriptions can be found in the documents $[3,16]$.

Off-axis configuration adds more difficulty to the fabrication and test of aspheric large mirrors. The primary mirror of GMT forms an ellipsoidal curve [17]. The surrounding six mirrors should have an identical off- axis aspheric shape, as shown in FIG. 2. The focal length of the primary mirror is 18.0 meters and the diameter is 25.4 meters. These make up f/0.7 focal ratio. The fast focal ratio contributes to the compactness of the GMT structure and reducing the size of the secondary mirror. The optical specifications of GMT are listed in TABLE 3.

As for testing the GMT off-axis aspheric primary mirrors, three methods have been devised. The first and main method is a phase-shift interferometry together with null correcting fold mirrors and CGH. It is a full-size high-resolution measurement of the optical figure. Though the test method needs a large area, there is a spatial restriction in the laboratory at The University of Arizona,

TABLE 3. Optical prescription of GMT.

\begin{tabular}{l|c|c}
\hline \hline Primary Mirror (M1) & segmented & Seven $(7) \times 8.4 \mathrm{~m}$ dia. Segments. \\
\hline Configuration & 25.448 meters & Non-circular aperture. Ellipse. \\
\hline Diameter, D1 & 36.000 meters & \\
\hline Radius of curvature, R1 & -0.99829 & \\
\hline Conic constant, K1 & 8.365 meters & Circular clear aperture. Off-axis segments tilted $13.522^{\circ}$ \\
\hline Segment diameter, $\mathrm{D}_{\mathrm{c}} 1$ & 1.78 meters & \\
\hline Center hole diameter & 7 segments & Conjugated with M1 segments \\
\hline Secondary Mirror $(\mathrm{M} 2)$ & 3.2 meters & Pupil stop. Non-circular aperture. \\
\hline configuration & 4.2058 meters & \\
\hline Diameter, D2 & -0.71087 & \\
\hline Radius of curvature, R2 & 1.063 meters & \\
\hline Conic constant, K2 & \multicolumn{2}{|c}{} \\
\hline Segment diameter, $\mathrm{D}_{\mathrm{c}} 2$ &
\end{tabular}




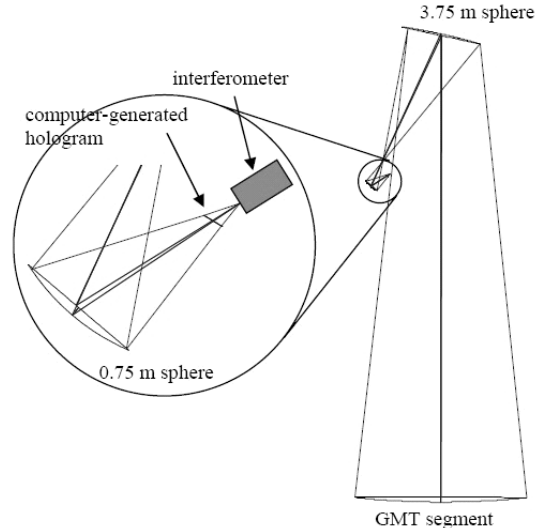

FIG. 3. Interferometry for the off-axis mirrors of the GMT Primary.

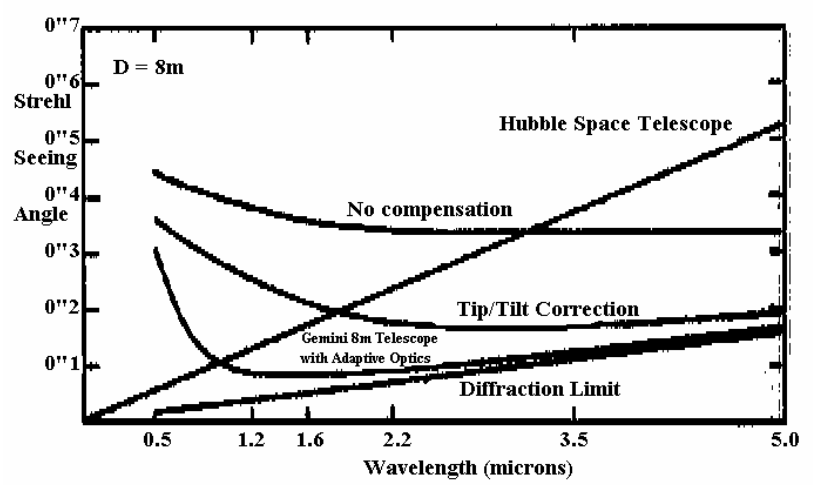

FIG. 4. Expected image quality of the Gemini telescopes [18].

where fabrication and test are conducted. Therefore a folding mirror of $3.75 \mathrm{~m}$ in diameter is adopted. Another $0.75 \mathrm{~m}$ folding mirror is also employed to reflect the rays to the interferometer. The folding spheres also act as null correctors, as illustrated in FIG. 3. Figure error of the $375 \mathrm{~m}$ sphere would bring large impact in the GMT test, so the sphere is also tested from its radius of curvature at the same time with the test of the GMT segment. And the error of the sphere is subtracted from the measurement of the large off-axis mirror.

The interferometry would be able to measure the figure and the geometry of the GMT segments to the required accuracy. However, a possibility of making a mistake in the performance of the test still exists. So it is necessary to have an independent measuring method, and a scanning pentaprism measurement is prepared. It measures slope errors in one-dimension across the mirror. A collimated laser beam is bent at 90 degrees and directed toward the off-axis test mirror by a pentaprism. The bent beam is set to be in parallel to the optical axis. The reflected beam by the off-axis test mirror is focused on the detector which sits at the focus, as the off-axis mirror is figured near parabolic. Deviations of the focused spot indicate slope errors on the mirror surface. The pentaprism moves linearly, and one-dimensional slope errors along one diameter can be measured.

The third method is a laser tracker measurement. This one is used when the mirrors are at the stages of grinding and initial polishing. It is a commercial device which combines a distance-measuring interferometer with angular encoders and servo-controlled pointing. The accuracy of the distance measurement is less than $1 \mu \mathrm{m}$ and that of angular encoder is about 1 arc-second, which are not accurate enough to measure large mirrors. However the method is still useful during the grinding and initial polishing, as it provides a quick look at the mirror surface, in about 15 minutes. Detailed description of the methods can be found in the Conceptual Design Review of GMT [17].

\section{SURFACE ACCURACY ASSESSMENT OF LARGE MIRRORS}

In order to test large aspheric mirrors, highly accurate test methods should be developed. In this chapter, required accuracy of testing devices is assessed. The Gemini telescopes are studied to find an appropriate accuracy of large mirrors. The Gemini telescopes are twin $8.1 \mathrm{~m}$ telescopes, located on Hawaii and in Chile, to observe both northern and southern hemispheres by using the identical telescopes. The primary mirror is $\mathrm{f} / 1.8$ meniscus mirror with a central hole of $1.2 \mathrm{~m}$ diameter.

The image quality was specified to be better than 0.1 arc second at near infrared wavelengths and near diffraction limit at shorter wavelengths with adaptive optics [18]. In the infrared bands, $50 \%$ of the energy will be concentrated within a circle of 0.1 arc second at the wavelength of $2.2 \mu \mathrm{m}$ [2]. In the optical region, $80 \%$ of encircled energy shall be fallen into 0.1 arc second at the wavelength of $550 \mathrm{~nm}$ [19]. In fact, the Gemini sites merely show median natural seeing of 0.4 arc seconds FWHM, and in the best condition the image quality is better than 0.25 arc seconds in near IR wavelength [20].

FIG. 4 [21] shows the expected image quality of the Gemini telescopes in several cases: no compensation, tip/ tilt correction, and adaptive optics. By applying adaptive optics, the image quality can reach the diffraction limit in the infrared band, and can be better than that of the Hubble Space Telescope at the wavelength of longer than $1 \mu \mathrm{m}$.

Three kinds of accuracy measures can be considered for the figure error of a mirror. They are slope error, rms (root-mean-square) surface error, and p-v(peak-tovalley) surface error. The tolerance of slope error can be simply calculated from the image size specified for the Gemini telescopes. In order for the image size to be 0.1 arc second, the primary mirror should have the slope error of $\pm 2.4 \times 10^{-7}$, if the secondary mirror is regarded as perfect. Therefore, the actual tolerance would be within the order of $10^{-7}$. 
TABLE 4. Relationship between Strehl ratio and rms wavefront error, and the difference.

\begin{tabular}{c|c|c}
\hline \hline Strehl ratio & RMS wavefront error $[\lambda]$ & Difference $[\lambda]$ \\
\hline 1.0 & 0 & \\
\hline 0.9 & 0.050 & 0.050 \\
\hline 0.8 & 0.071 & 0.021 \\
\hline 0.7 & 0.087 & 0.016 \\
\hline
\end{tabular}

The rms wavefront error can be converted from Strehl ratio. Between the rms error and Strehl ratio, there is an approximate relationship as shown [22]:

Strehl ratio $\sim 1-4 \pi^{2}(\mathrm{rms} / \lambda)^{2}$,

where $\lambda$ is the wavelength to observe and rms is rms wavefront error. Precise telescope optics normally require the Strehl ratio to be about 0.8 [22]. The Gemini telescope is also required to be 0.8 of the Strehl ratio at the wavelength of $1.6 \mu \mathrm{m}$ [23]. When the Strehl ratio is 0.8 , the rms wavefront error is $0.07 \lambda$. TABLE 4 shows the relationship between Strehl ratio and rms wavefront error. The last column, 'Difference', means the difference of the rms at every 0.1 change of the Strehl ratio. The difference value is about $0.02 \lambda$ or more in the interval of 0.1 in Strehl ratio. The wavefront error is half the height error on a mirror surface. Therefore, if a testing method has the accuracy of about $0.01 \lambda$ in rms height error, the Strehl ratio of the tested optics would have the accuracy of 0.1 .

Concerning the accuracy of peak-to-valley (p-v), a useful data can be found at COSTAR (Corrective Optics Space Telescope Axial Replacement) in Hubble Space Telescope. The mirrors achieved $\lambda / 8$ in $\mathrm{p}-\mathrm{v}$ wavefront error on each Zernike term, where $\lambda$ is the wavelength of $632.8 \mathrm{~nm}$ [5]. The tolerance of $\mathrm{p}-\mathrm{v}$ surface error can simply be estimated to be $\lambda / 20(0.05 \lambda)$, which is less than half the $\mathrm{p}-\mathrm{v}$ of COSTAR. As a rule of thumb, rms is assumed to be one fourth or one fifth of $\mathrm{p}-\mathrm{v}$ [24]. Determining the measuring accuracy of $\mathrm{p}-\mathrm{v}$ by $0.05 \lambda$ seems adequate when rms is measured to an accuracy of $0.01 \lambda$. The primary mirror of Gemini has the surface accuracy of about $15 \mathrm{~nm}$ rms. That is about $0.03 \lambda$ at the wavelength of $500 \mathrm{~nm}$, which is sensible and matches the above assessments nearly.

\section{RESULTS AND DISCUSSIONS}

As Astronomy and opto-mechanical technology become advanced, larger telescopes have been developed. From last decade, more than a dozen $8 \mathrm{~m}$ class telescopes have been constructed and being operated. As the size of mirror becomes larger, the accuracy is required to be higher reaching up to the diffraction limit. Now next generation telescopes of $30 \mathrm{~m}$ class are being designed, with expected completion by next decade. GMT contains seven $8.4 \mathrm{~m}$ aspheric mirror segments as a primary mirror. Six of the GMT primary mirrors are off-axis aspheric, which is more difficult to test. Three test methods for the offaxis aspheric mirrors are reviewed.

Required accuracy of the test methods is assessed for the large aspheric mirrors. Gemini $8.1 \mathrm{~m}$ telescopes are selected as a case study. Three kinds of measuring entities are considered, which are slope error, p-v error, and rms error. Measuring devices would be good to have slope error within the order of $10^{-7}$, p-v error to an accuracy of $0.05 \lambda$, and rms error about $0.01 \lambda$. Test methods should be more accurate than the surface accuracy of the mirror to be produced.

Such test methods are also widely applied to other applications, such as wafer inspection, testing camera system, 3-D reconstruction, etc. [25, 26, 27]

\section{REFERENCES}

1. A. Isaacs, E. Martin, J. Law, and F. Alexander, The Macmillan Compact Encyclopedia (Macmillan Publishers Ltd., London, UK, 1994).

2. F. Gillett and M. Mountain, "A scientific overview," The Gemini Newsletter 12, 10-18 (1996).

3. Y.-S. Kim, "An improved geometric test for optical surfaces," Ph.D. Thesis, London University (1998).

4. L. Furey, T. Dubos, D. Hansen, and J. Samuels-Schwarz, "Hubble Space Telescope primary-mirror characterization by measurement of the reflective null corrector," Appl. Opt. 32, 1703-1714 (1993).

5. L. Feinberg and M. Wilson, "Hubble Space Telescope COSTAR asphere verification with a modified computergenerated hologram interferometer," Appl. Opt. 32, 17861788 (1993).

6. J. H. Burge, "Measurement of large convex aspheres," Proc. SPIE 2871, 362-373 (1997).

7. Y.-Y. Cheng and J. C. Wyant, "Two-wavelength phase shifting interferometry," Appl. Opt. 23, 4539-4543 (1984).

8. K. Creath, Y.-Y. Cheng, and J. C. Wyant, "Contouring aspheric surfaces using two-wavelength phase-shifting interferometry," Optica Acta 32, 1455-1464 (1985).

9. J. Kandulla, B. Kemper, S. Knoche, and G. Bally, "Twowavelength method for endoscopic shape measurement by spatial phase-shifting speckle-interferometry," Appl. Opt. 43, 5429-5437 (2004).

10. Y. Bitou, "Two-wavelength phase-shifting interferometry with a superimposed graing displayed on an electrically addressed spatial light modulator," Appl. Opt. 44, 15771581 (2005).

11. D. S. Mehta, S. K. Dubey, M. M. Hossain, and C. Shakher, "Simple multifrequency and phase-shifting fringe-projection system based on two-wavelength lateral shearing interferometry for three-dimensional profilometry," Appl. Opt. 44, 7515-7521 (2005).

12. J. G. Dil, W. Mesman, and J. C. Driessen, "High-precision 
measurement of aspheric surfaces," Proc. SPIE 235, 8590 (1980).

13. M. Melozzi, L. Pezzati, and A. Mazzoni, "Testing aspheric surfaces using multiple annular interferograms," Opt. Eng. 32, 1073-1079 (1993).

14. X. Hou, F. Wu, B. Lei, B. Fan, and Q. Chen, "Testing large aspheric surfaces with complementary annular subaperture interferometric method," Proc. SPIE 7018, 70183K1-70183K-8 (2008).

15. J. H. Burge, P. Su, and C. Zhao, "Optical metrology for very large convex aspheres,” Proc. SPIE 7018, 7018181-701818-12 (2008).

16. Y.-S. Kim, "Developing an optical testing method for space telescopes," J. Opt. Soc. Korea 5, 33-36 (2001).

17. GMTO, Giant Magellan Telescope Conceptual Design Review (Pasadena, CA, USA, 2006).

18. P. S. Osmer, Gemini science requirements, version 1.1 (1992).

19. E. Hansen, "Gemini telescopes f/16 optical design summary," in Gemini 8-M Telescopes Project Report, RPTO-G0047 (Gemini Project Office, Tucson, AZ, USA, 1994).
20. P. Puxley, "Classical and queue scheduling of the gemini telescopes," The Gemini Newsletter 13, 10-16 (1996).

21. M. Mountain, "What is beyond the current generation of groundbased $8 \mathrm{~m}-10 \mathrm{~m}$ class telescopes and the VLT-I?," Proc. SPIE 2871, 597-606 (1997).

22. M. Born and E. Wolf, Principles of Optics, 5th ed. (Pergamon, Oxford, UK, 1975).

23. J. Oschmann and D. A. Simons, "Gemini 8 meter telescopes performance estimates update," Gemini Preprint 6, 1-12 (1996).

24. W. J. Smith, Modern Optical Engineering (McGraw-Hill Book Co., New York, USA, 1990).

25. J. H. Lee, Y. Kim, J. Kim, and Y. Yoo, "Bare wafer inspection using a knife-edge test,” J. Opt. Soc. Korea 11, 173-176 (2007).

26. H.-J. Yang, J.-S. Cho, and Y.-H. Won, "Reduction of reconstruction errors in kinoform CGHs by modified simulated annealing algorithm,” J. Opt. Soc. Korea 13, 92-97 (2009).

27. D. Kim and Y. J. Cho, "3-D surface profile measurement using an acousto-optic tunable filter based spectral phase shifting technique, J. Opt. Soc. Korea 12, 281-287 (2008). 